\title{
Role of gefitinib in the targeted treatment of non- small-cell lung cancer in Chinese patients
}

This article was published in the following Dove Press journal:

OncoTargets and Therapy

9 March 2016

Number of times this article has been viewed

\author{
Meng-Jiao Li \\ Qing $\mathrm{He}$ \\ Mei Li \\ Feng Luo \\ Yong-Song Guan \\ Department of Oncology, Center \\ of Oncology, West China Hospital \\ of Sichuan University, Chengdu, \\ People's Republic of China
}

Correspondence: Yong-Song Guan Center of Oncology, West China Hospital of Sichuan University, Chengdu 61004I, People's Republic of China Email yongsongguan@yahoo.com

\begin{abstract}
Non-small-cell lung cancer (NSCLC) is the most common type of lung cancer. Conventional treatment options have limited efficacy because most cases are in the advanced stage at the time of diagnosis. In recent years, gefitinib, an epidermal growth factor receptor tyrosine kinase inhibitor, has shown its good antitumor activities in treating NSCLC in a number of studies. This paper reviews its role in the targeted treatment of NSCLC in Chinese patients.
\end{abstract}

Keywords: pulmonary carcinoma, therapy, EGFR-TK inhibitor, status, People's Republic of China

\section{Introduction}

Lung cancer is a very common and highly malignant carcinoma worldwide. It is still the first cause of cancer-related deaths around the world with $\sim 1.3$ million deaths per year. ${ }^{1}$ The incidence and mortality rates of lung cancer in Chinese cancer registration areas were 46.08/100,000 and 37.00/100,000, respectively, in 2010. ${ }^{2}$ Furthermore, the incidence increases rapidly at an annual rate of $26.9 \%$ and the total cases are expected to reach 100 million until 2025 when the People's Republic of China might become the country with the largest number of lung cancer patients.

Lung cancer is classified into the following two basic types: small-cell lung cancer and non-small-cell lung cancer (NSCLC), according to the histological types published by the World Health Organization, with $80 \%-85 \%$ being NSCLC. ${ }^{3}$ For many years, therapeutic strategies for lung cancer have been focused on traditional therapy, such as surgery, chemotherapy, and radiotherapy. The treatment of early stage lung cancer usually gives priority to surgery. Unfortunately, $\sim 70 \%-80 \%$ of the patients have been in the advanced stage at the time of diagnosis and lost the chance for surgery due to lack of symptoms in early NSCLC. ${ }^{4}$ Standard first-line systemic treatment for advanced NSCLC consisted of chemotherapy with a combination of two drugs, including a platinum compound and a nonplatinum drug, such as pemetrexed, gemcitabine, vinorelbine, or a taxane. ${ }^{5}$ However, platinum-based doublet chemotherapy still had limited efficacy because median survival was only 12-13 months and 1-year survival rate was 50\%-55\%. ${ }^{6}$ Other therapeutic procedures include radiotherapy and complementary therapies in palliative and hospice care, usually with disappointing results. ${ }^{3,7,8}$ Moreover, the therapeutic methods for elderly patients with NSCLC and patients with poor performance status are always limited because these patients are not eligible for aggressive therapies due to the age-related reduction in the functional reserve of many organs and the presence of comorbidities. ${ }^{3,8}$ IFCT-0501, the largest Phase III randomized trial dedicated to elderly patients with advanced NSCLC, demonstrated only a 6.2-month median overall survival (OS) and 25.4\% 1-year survival for vinorelbine or gemcitabine monotherapy in elderly patients. Although the median OS and 1-year 
survival for carboplatin plus paclitaxel-doublet chemotherapy were improved to 10.3 months and $44.5 \%$, respectively, an increase in toxic effects was observed in these patients. ${ }^{9}$ The treatment of NSCLC requires advancement urgently.

In recent years, with the growth of knowledge in driver gene mutations and signal transduction, new targeted therapies for different cell surface molecules are constantly emerging. ${ }^{3}$ The most widely recognized genomic alterations include epidermal growth factor receptor $(E G F R)$ mutations, which have been recorded more frequently in Asian than in non-Asian patients, ${ }^{10,11}$ and echinoderm microtubuleassociated protein-like 4-anaplastic lymphoma kinase rearrangements. ${ }^{12}$ The discovery of new targeted drugs, such as EGFR tyrosine kinase inhibitors (EGFR-TKIs) (gefitinib, erlotinib, and afatinib) and anaplastic lymphoma kinase inhibitors (crizotinib and ceritinib), has significantly changed the treatment in this setting. As the most important kind of targeted drugs, EGFR-TKI drugs occupy an irreplaceable position in this field. Gefitinib (Iressae, ZD1839; AstraZeneca plc, London, UK), the first targeted drug used for NSCLC patients, is an orally active and highly effective EGFR tyrosine kinase (TK) selective inhibitor that blocks signal transduction pathways implicated in cancer growth. In both in vitro and in vivo studies, gefitinib has shown perfect antitumor activities in treating NSCLC. ${ }^{13}$

\section{Epidermal growth factor receptor}

The human EGFR (HER) family contains four members: EGFR (otherwise known as HER1), HER2, HER3, and HER4, all belonging to the TK family of proteins. EGFR, one kind of multifunctional glycoproteins widely expressed in normal tissues and neoplastic lesions of most organs, plays a crucial role in cell proliferation and differentiation. ${ }^{14,15}$ The important discovery that the avian erythroblastic leukemia viral (v-erb-b) oncogene is the active variant of EGFR derivative has proved that EGFR and the HER family are often associated with cancer initially. ${ }^{16}$ Research shows that EGFR and its ligand are part of the cell signal transduction system, and its signal transduction network accounts for an important position in the process of formation and development of tumors. Changes in expression and aberrant activation of EGFR have been verified to be associated with various cancers. ${ }^{15}$

The human EGFR gene, with a total length of $200 \mathrm{~kb}$, is located in the chromosome 7 p13-q22 area, consisting of 28 exons and coding 1,186 amino acids. EGFR protein, a $170 \mathrm{kDa}$ plasma membrane glycoprotein, is composed of an extracellular domain, a single hydrophobic transmembrane region, an intracellular portion that contains the
C-terminal tail, and a TK domain that is important for signal transduction. ${ }^{17}$ Upon ligand binding, a receptor TK undergoes a series of changes, the earliest one being the conversion of the monomeric form of the receptor to the dimeric form. This leads to activation of the kinase, resulting in phosphorylation of its own tyrosine residues, a phenomenon known as autophosphorylation. The phosphotyrosine residues of the activated receptor then act as docking sites for target molecules, such as signal transducers. This association triggers off signaling cascades through pathways such as PI3-kinase-AKT, RAS/RAF, and PI3K-Akt and modulates cell proliferation, survival, adhesion, migration, and differentiation. ${ }^{18}$

\section{EGFR tyrosine kinase inhibitor}

At present, EGFR-targeted drugs contain a monoclonal antibody, such as cetuximab, that targets the extracellular domain of EGFR and the small-molecule EGFR TK selective inhibitor that targets the TK domain in the intracellular portion and therefore blocks the signal transduction pathways implicated in the proliferation and survival of cancer cells. ${ }^{19}$

The EGFR protein is overabundant in $\sim 40 \%-80 \%$ of NSCLC tumors. ${ }^{20}$ Initially, investigators believed that gefitinib may completely terminate EGFR activity, which plays a pivotal role in the management of cellular growth and proliferation. However, the clinical trials were disappointing because tumor response was not corresponding to the amount of EGFR. ${ }^{21}$ In June 2004, researchers at Harvard Medical School first reported that specific mutations in the TK domain of the EGFR gene may be the necessary precondition of clinical responsiveness to the TKI. ${ }^{22}$ Analysis showed that the response to gefitinib was $>80 \%$ in tumors with positive mutations in the TK domain of the EGFR gene. On the contrary, little effect was found in tumors with negative mutations. Once published, these significant research findings created high attention from scholars all over the world and were continuously identified in the USA, Japan, Korean, and the People's Republic of China after $<1$ year. Researchers from Taiwan first illustrated the association between EGFR mutation status of tumors and the responsiveness to gefitinib in Chinese people. Among the 16 patients treated with gefitinib, seven of nine who responded had EGFR mutations, while only one of seven who failed to respond had EGFR mutations. The difference in mutation rates between the responders and nonresponders was statistically significant $\left(P<0.041\right.$, by $\chi^{2} /$ Fisher's exact tests $){ }^{23}$

The EGFR gene is composed of 28 exons, and the entire TK domain is encoded by exons $18-24$. So far, $\sim 90 \%$ of EGFR gene mutations were discovered in exons 19-21. 
Specific $E G F R$-activating mutations are either short, in-frame nucleotide deletions, in-frame insertions/duplications, or single-nucleotide replacements gathered around the adenosine triphosphate (ATP)-binding area of the TK domain. ${ }^{24}$ To date, in-frame deletions in exon 19 around the LeuArgGluAla motif(del19) at residues 746-750 (the most common being del E746-A750) and exon 21 Leu858Arg (L858R) point mutation are the best characterized mutations, together representing $85 \%-90 \%$ of all EGFR mutations in NSCLC. ${ }^{24}$ Other EGFR-activating mutations have been described and are usually defined as "other uncommon mutations." However, their ability to predict the sensitivity to a reversible EGFR-TKI is less distinct compared with the del19 or L858R mutation. ${ }^{25}$

In the investigation on EGFR gene mutations, the difference between the People's Republic of China and the world advanced level was only 6 months because of the research directed by Taiwan experts. ${ }^{23}$ A related article from mainland China was published in March 2005 that identified ten somatic mutations from a total of 41 lung cancer patients in the People's Republic of China. ${ }^{26}$ Similar to the result analysis of overseas data, eight of these mutations are deletions in exon 19, one point mutation in exon 21 , and one deletion/insertion in exon 20. Based on the data of 76 lung cancer patients from mainland China completed by Beijing Union Medical College investigators, a research on the detection of EGFR gene mutation first showed that there was a distinct correlation between EGFR mutation conditions and responsiveness to gefitinib in mainland Chinese patients with NSCLC. ${ }^{27}$

\section{Pharmacokinetics}

Gefitinib is an anilinoquinazoline compound with the chemical name of 4-quinazolinamine, $N$-(3-chloro-4-flurophenyl)7-methoxy-6-[3-(4-morpholinyl)propoxy]. The molecular formula is $\mathrm{C} 22 \mathrm{H} 24 \mathrm{ClFN} 4 \mathrm{O} 3$. The maximum plasma concentration of gefitinib appears within 3-7 hours after a $250 \mathrm{mg}$ oral dose. ${ }^{28}$ Gefitinib is extensively distributed into the tissues and highly protein bound (to albumin and $\alpha 1$-acid glycoprotein), which results in a large volume of distribution of 1,400 L and a long half-life of 48 hours, retaining a stable blood drug concentration for 7-10 days. ${ }^{29}$ It has been observed that the absolute bioavailability is $60 \%$, similar to that in healthy volunteers and cancer patients. ${ }^{30}$ Irrespective of the administration route, including a granular formulation, a dispersion of the classic tablets, or administration by nasogastric tube, there was no significant influence on its bioavailability. ${ }^{31,32}$
Studies have indicated that $\mathrm{AUC}_{0-\infty}$ and $C_{\max }$ exhibited linear kinetics over the therapeutic dosing range between 50 and $500 \mathrm{mg}$; with doses $\geq 800 \mathrm{mg}$, and $45 \%$ of patients required dose reductions. Bergman et al observed that the AUC and $C_{\max }$ exhibited obvious individual differences, which reached as high as 15 times. They believed that the huge differences were caused by gefitinib exposure in the plasma, which was influenced by the polymorphism of enzyme, transport protein, and many other factors. ${ }^{33}$ In addition, the expected steady-state blood concentrations of medicine valley were not associated with patients' age, weight, sex, ethnicity, or creatinine clearance rate according to a population-based data analysis in tumor patients. ${ }^{34}$

Gefitinib is extensively metabolized in the liver, predominantly by cytochrome P450 (CYP) 3A4. An in vivo study aimed to examine the kinetics of metabolism of gefitinib and erlotinib by individual CYP enzymes elucidated that gefitinib was metabolized by CYP3A4, CYP3A5, CYP2D6, and CYP1A1 and to a negligible extent by CYP1A2 and CYP1B1. ${ }^{35}$ The identified metabolic pathways include $O$-demethylation, dealkylation, and oxidative defluorination. There are five metabolites identified in plasma; the $O$-desmethyl derivative, the major metabolite, has EGFR-TK inhibition activity in an enzyme assay comparable with that of the parent drug. However, the metabolite has lower activity in a cell-based assay due to the poor penetration into the cell and is therefore unlikely to contribute significantly to the therapeutic activity. Approximately $90 \%$ of gefitinib is recovered in feces $(86 \%)$ and urine $(0.5 \%)$ in over 10 days. ${ }^{36}$

\section{Large clinical studies}

Multiple clinical studies have demonstrated that gefitinib has notable curative effectiveness for NSCLC. It has been approved for second- or third-line therapy of advanced or metastatic NSCLC and the first-line therapy for patients with EGFR mutation-positive tumors.

Phase I trials have identified that the optimal dose range of safety is $250-500 \mathrm{mg} / \mathrm{d}$ that has been confirmed by dose escalation safety/tolerability trials. Studies have shown that $250 \mathrm{mg} / \mathrm{d}$ was the minimum concentration for effective treatment and high tolerance, ensuring the inhibition of EGFR signal transduction, which was detected through skin biopsy, and $500 \mathrm{mg} / \mathrm{d}$ was the highest dose level tolerated by most patients in long-term treatment. ${ }^{37,38}$

Iressa Dose Evaluation in Advanced Lung Cancer (IDEAL) I and II, ${ }^{39,40}$ two randomized, double-blind, parallelgroup, multicenter Phase II trials of gefitinib at 250 and $500 \mathrm{mg} / \mathrm{d}$, which followed the results in previous studies, 
investigated the efficacy and safety of gefitinib at 250 and $500 \mathrm{mg} / \mathrm{d}$ in patients with advanced NSCLC who had previously received one or two chemotherapy regimens. ${ }^{37,38}$ The two trials demonstrated that both 250 and $500 \mathrm{mg} / \mathrm{d}$ doses of gefitinib were equally active in an EGFR mutation-unselected patient population, while the higher dose was associated with greater frequency and severity of adverse events (AEs), resulting in response rates of $\sim 20 \%$ and median progressionfree survival (PFS) of 2.7 and 2.8 months for the 250 and $500 \mathrm{mg} / \mathrm{d}$ doses of gefitinib, respectively. ${ }^{39,40}$ The results of the study mentioned earlier laid the status of gefitinib treatment of advanced NSCLC. Interestingly, a subset of patients treated with gefitinib demonstrated a very positive response. A retrospective analysis of IDEAL I showed that in Japanese patients, 250 and $500 \mathrm{mg} / \mathrm{d}$ of gefitinib were associated with median survival periods of 13.8 and 11.2 months, respectively, compared with 7.6 and 8.0 months, respectively, in the overall population. ${ }^{41}$ At the time, the implications of EGFR mutations were not understood, but we now know that it is because of Asian ethnicity having a higher EGFR gene mutation rate.

Iressa Survival Evaluation in Lung Cancer (ISEL) was a double-blind, placebo-controlled, parallel-group, multicenter, randomized, Phase III study that assessed the survival advantage of gefitinib $(250 \mathrm{mg} / \mathrm{d})$ as second- or third-line treatment for patients with locally advanced or metastatic NSCLC, undertaken in 210 centers in 28 countries across Europe, Asia, Central and South America, Australia, and Canada. ${ }^{42}$ Unlike IDEAL I/II, this study failed to meet its end point of improved OS, with a median survival of 5.6 months for patients treated with gefitinib as compared with 5.1 months for patients treated with placebo (hazard ratio $[\mathrm{HR}]=0.89,95 \% \mathrm{CI}: 0.77-1.02, P=0.087)$. This led to the restriction of gefitinib use in June 2005 by the US Food and Drug Administration (FDA) and to the withdrawal of the marketing authorization application from AstraZeneca, which was under review in Europe. However, preplanned subgroup analyses showed significantly longer survival in the gefitinib group than that in the placebo group in never-smokers ( $n=375 ; 0.67$ [0.49-0.92], $P=0.012$; median survival 8.9 vs 6.1 months) and in patients of Asian origin ( $\mathrm{n}=342 ; 0.66$ [0.48-0.91], $P=0.01$; median survival $9.5 \mathrm{vs}$ 5.5 months $).{ }^{43}$

INTEREST is the first Phase III study that compared OS of gefitinib-treated patients with that of docetaxel-treated patients by coprimary analyses of noninferiority in the overall population in pretreated NSCLC ( $\geq$ one platinumbased regimen). The analysis confirmed the noninferiority of gefitinib compared with docetaxel in OS (593 vs 576 events, $\mathrm{HR}=1.020$, 95\% CI: 0.905-1.150); 1-year survival rates were $32 \%$ and $34 \%$, and median survival periods were 7.6 and 8.0 months, respectively. Moreover, gefitinib was well tolerated and less toxic than chemotherapy. The researchers also compared gefitinib with docetaxel in the subgroup of 174 patients with high EGFR gene copy number, but the superiority of gefitinib in patients with high EGFR gene copy number was not proven ( 72 vs 71 events, $\mathrm{HR}=1.09,95 \% \mathrm{CI}$ : $0.78-1.51, P=0.62$; median survival 8.4 vs 7.5 months). ${ }^{44}$

A meta-analysis evaluated the efficacy and safety of gefitinib and standard second-line chemotherapy for the treatment of NSCLC and showed that both PFS and overall response rate (ORR) were better in the gefitinib group than in the chemotherapy group. As a second-line therapy for advanced NSCLC, gefitinib has exhibited excellent efficacy and high tolerance. ${ }^{45}$

First-line treatment for NSCLC with gefitinib has also undergone continuous exploration. One of the most representative studies is IPASS, a Phase III, randomized, open-label, first-line study of gefitinib vs carboplatin/paclitaxel in clinically selected patients with advanced NSCLC in Asia. ${ }^{46}$ IPASS selectively recruited Asian patients who were previously untreated, never-smokers, or light ex-smokers with advanced pulmonary adenocarcinoma based on the identification of clinical characteristics that predicted the response to gefitinib relative to placebo in the ISEL study. The study met its primary objective end point, showing noninferiority of gefitinib in terms of OS and superiority in ORR and PFS compared with carboplatin/paclitaxel chemotherapy. In the subgroup analyses, it exhibited significant differences in PFS and ORR between the gefitinib group and the chemotherapy group among EGFR mutation-positive patients (PFS 9.5 vs 6.3 months, $\mathrm{HR}=0.48, P=0.001$; ORR: $71.2 \%$ vs $47.3 \%$, odds ratio: $2.75, P=0.0001)$. This research confirmed that EGFR mutation was the strongest predictive biomarker for the effect of EGFR-TKIs in advanced NSCLC. Gefitinib became an option for first-line standard of care for NSCLC patients with EGFR-mutated tumor.

It is inconclusive for clinicians to choose to suspend EGFR-TKI treatment and begin platinum-based chemotherapy or a combined use of EGFR-TKI and platinum-based chemotherapy after disease progression. IMPRESS ${ }^{47}$ is the first and only randomized Phase III study that evaluated the efficacy/safety of continuing gefitinib plus cisplatin/ pemetrexed (cis/pem) (G) vs placebo plus cis/pem (P) in patients with acquired resistance to first-line gefitinib. 
Table I Summary of large clinical studies on gefitinib

\begin{tabular}{|c|c|c|c|c|c|c|c|}
\hline Study & Phase & $\begin{array}{l}\text { Population } \\
\text { (patients, n) }\end{array}$ & EGFR status & Treatment & ORR (\%) & $\begin{array}{l}\text { Median PFS } \\
\text { (months) }\end{array}$ & $\begin{array}{l}\text { Median OS } \\
\text { (months) }\end{array}$ \\
\hline \multicolumn{8}{|c|}{ Second- or third-line treatment (patients had failed previous chemotherapy) } \\
\hline IDEAL I ${ }^{39}$ & II & $\begin{array}{l}\text { Unselected } \\
(n=210)\end{array}$ & Unknown & $\begin{array}{l}\text { Gefitinib } \\
\text { (250 vs } 500 \mathrm{mg} \text { ) }\end{array}$ & 18.4 vs 19.0 & 2.7 vs 2.8 & 7.6 vs 8.0 \\
\hline IDEAL $2^{40}$ & II & $\begin{array}{l}\text { Unselected } \\
(n=22 I)\end{array}$ & Unknown & $\begin{array}{l}\text { Gefitinib } \\
\text { (250 vs } 500 \mathrm{mg} \text { ) }\end{array}$ & 12.0 vs 9.0 & - & 7.0 vs 6.0 \\
\hline ISEL ${ }^{42}$ & III & $\begin{array}{l}\text { Unselected } \\
(n=1,692)\end{array}$ & Unknown & Gefitinib vs placebo & 8.0 vs 1.3 & 3.0 vs 2.6 & 5.6 vs 5.1 \\
\hline INTEREST ${ }^{44}$ & III & $\begin{array}{l}\text { Unselected } \\
(n=I, 466)\end{array}$ & $\begin{array}{l}\text { High EGFR gene } \\
\text { copy ( } n=85 \text { vs } 89)\end{array}$ & $\begin{array}{l}\text { Gefitinib }(250 \mathrm{mg}) \text { vs } \\
\text { docetaxel }\left(75 \mathrm{mg} / \mathrm{m}^{2}\right)\end{array}$ & 9.1 vs 7.6 & 2.2 vs 2.7 & 7.6 vs 8.0 \\
\hline \multicolumn{8}{|c|}{ Second-line treatment (patients with acquired resistance to first-line gefitinib) } \\
\hline IMPRESS ${ }^{47}$ & III & $\begin{array}{l}\text { Unselected } \\
(n=265)\end{array}$ & EGFR-M(+) & $\begin{array}{l}\text { Gefitinib }(250 \mathrm{mg}) \text { vs } \\
\text { cisplatin/pemetrexed }\end{array}$ & & 5.44 vs 5.4 & $\begin{array}{l}\text { Immature (33\% of } \\
\text { patients had died) }\end{array}$ \\
\hline \multicolumn{8}{|c|}{ First-line treatment } \\
\hline IPASS $^{46}$ & III & $\begin{array}{l}\text { Asia, untreated, } \\
\text { never-smokers or } \\
\text { light ex-smokers, } \\
\text { adenocarcinoma } \\
(n=1,2 \mid 7)\end{array}$ & $\begin{array}{l}\text { EGFR-M(+) } \\
(n=262) \\
\text { EGFR-M(-) } \\
(n=176) \\
\text { Unknown }(n=780)\end{array}$ & $\begin{array}{l}\text { Gefitinib }(250 \mathrm{mg}) \\
\text { vs carboplatin }+ \\
\text { paclitaxel }\end{array}$ & 43.0 vs 32.2 & 5.6 vs 5.7 & 18.8 vs 17.4 \\
\hline
\end{tabular}

Abbreviations: EGFR, epidermal growth factor receptor; ORR, overall response rate; PFS, progression-free survival; OS, overall survival; M, mutation.

Results showed that there was no statistically significant improvement in PFS for G vs P (median PFS 5.4 months each) and OS was immature (33\% of patients died). No treatment differences were found in ORR/disease control rate (DCR). The researchers indicated that continuation of gefitinib in addition to cis/pem would be of no clinical benefit for patients with acquired resistance to gefitinib. Thus, the standard of care should remain as doublet chemotherapy alone. The study explored the confusing problem of clinical application of targeted drugs, and the outcome would be conducive to doctors' clinical practice. Table 1 summarizes the large clinical studies on gefitinib.

\section{Clinical application of gefitinib in Chinese patients with NSCLC}

The efficacy of gefitinib has been explored extensively in the West and in Japan, and similar studies have highlighted the clinical benefit of the use of gefitinib in patients with advanced NSCLC in Asian origin. A previous study from Singapore in Chinese patients also conducted subgroup analyses, which showed that patients with clinical characteristics, such as Asian ethnicity, female sex, nonsmoking status, and adenocarcinomas, were associated with a higher likelihood of response to several treatments. ${ }^{48}$ The People's Republic of China possesses the largest number of lung cancer cases compared to all over the world. Table 2 highlights the clinical application and research situation of gefitinib.

\section{Gefitinib as a therapy for advanced NSCLC}

As early as the time when gefitinib was approved by the FDA for the treatment of NSCLC after chemotherapy failure, Chinese clinical experts had designed a trial to evaluate the efficacy of gefitinib in Chinese patients with locally advanced or metastatic NSCLC after failure of previous chemotherapy and began to explore its safety according to the State FDA from November 2003 to June 2004. ${ }^{49}$ Totally, 159 patients were recruited from Guangzhou, Beijing, Shanghai, and other places to eight clinical medical centers, and at least one treatment of $250 \mathrm{mg} / \mathrm{d}$ of gefitinib was administered until the objective evidence of disease progression or intolerable AEs was observed. The outcome demonstrated DCR of $54.1 \%$, ORR of $27.5 \%$, PFS of 10.0 months, and 1-year survival rate of $44 \%$, consistent with other large-scale clinical studies. The trial verified that gefitinib had obvious curative effect and could become a new advantageous treatment option for Chinese patients with locally advanced or metastatic NSCLC. Based on the previous results and data analysis of IDEAL, the State FDA approved gefitinib for the treatment of locally advanced or metastatic NSCLC after failure of previous chemotherapy in the People's Republic of China on February 25, 2005.39,49

Expanded access program (EAP), a global compassionateuse program implemented by AstraZeneca starting in 2000, was conducted to provide charitable gefitinib therapy 
Table 2 Clinical studies on gefitinib in Chinese patients with NSCLC

\begin{tabular}{|c|c|c|c|c|c|c|c|}
\hline Study & Phase & Population (patients, $\mathrm{n}$ ) & EGFR status & Treatment & ORR (\%) & Median PFS & Median OS \\
\hline \multicolumn{8}{|c|}{ Second- or third-line treatment } \\
\hline Guan et $\mathrm{a}^{49}$ & III & Unselected $(n=159)$ & Unknown & Gefitinib (250 mg) & 27.0 & 97 days & $10.0 \mathrm{~m}$ \\
\hline Huang et $\mathrm{al}^{50}$ & III & Unselected $(n=103)$ & Unknown & Gefitinib (250 mg) & 18.4 & $3 \mathrm{~m}$ & $9.8 \mathrm{~m}$ \\
\hline Zhang et $\mathrm{al}^{51}$ & II & Unselected $(n=98)$ & $\begin{array}{l}\text { EGFR-M(+) }(n=12) \\
\text { EGFR-M(-) }(n=18) \\
\text { Unknown }(n=68)\end{array}$ & Gefitinib (250 mg) & 31.6 & $7.0 \mathrm{~m}$ & $12.0 \mathrm{~m}$ \\
\hline \multicolumn{8}{|c|}{ First-line treatment } \\
\hline Wu et $\mathrm{al}^{52}$ & $\begin{array}{l}\text { Further } \\
\text { analysis } \\
\text { of IPASS }\end{array}$ & $\begin{array}{l}\text { Chinese, untreated, never-smokers } \\
\text { or light ex-smokers, adenocarcinoma } \\
(n=372)\end{array}$ & $\begin{array}{l}\text { EGFR-M(+) }(n=39) \\
\text { EGFR-M(-) }(n=48) \\
\text { Unknown }(n=285)\end{array}$ & $\begin{array}{l}\text { Gefitinib }(250 \mathrm{mg}) \\
\text { vs carboplatin }+ \\
\text { paclitaxel }\end{array}$ & 46.6 vs 29.8 & 6.8 vs $6.8 \mathrm{~m}$ & 18.1 vs $18.3 \mathrm{~m}$ \\
\hline Lou et $\mathrm{al}^{53}$ & III & $\begin{array}{l}\text { Chinese, untreated, never-smokers } \\
\text { or light ex-smokers, adenocarcinoma } \\
(n=5 I)\end{array}$ & $\begin{array}{l}\text { EGFR-M(+) }(n=2) \\
\text { EGFR-M(-) }(n=5) \\
\text { Unknown }(n=44)\end{array}$ & $\begin{array}{l}\text { Gefitinib }(250 \mathrm{mg}) \\
\text { vs carboplatin }+ \\
\text { paclitaxel }\end{array}$ & 36.6 vs 42.3 & $4.2 \mathrm{~m}$ vs $8.3 \mathrm{~m}$ & $14.4 \mathrm{~m}$ vs $15.0 \mathrm{~m}$ \\
\hline Li et al ${ }^{54}$ & & Unselected $(n=54)$ & EGFR-M(+) & Gefitinib (250 mg) & 90.0 & $8.3 \mathrm{~m}$ & $19.5 \mathrm{~m}$ \\
\hline \multicolumn{8}{|c|}{ Maintenance treatment } \\
\hline INFORM $^{58}$ & III & $\begin{array}{l}\text { First-line chemotherapy without PD } \\
(\mathrm{n}=296)\end{array}$ & $\begin{array}{l}\text { EGFR-M(+) }(n=30) \\
\text { EGFR-M(-) }(n=49) \\
\text { Unknown }(n=217)\end{array}$ & $\begin{array}{l}\text { Gefitinib } \\
(250 \mathrm{mg}) \text { vs } \\
\text { placebo }\end{array}$ & 26.0 vs 21.0 & 4.8 vs $2.6 \mathrm{~m}$ & $18.7 \mathrm{~m}$ vs $16.9 \mathrm{~m}$ \\
\hline \multicolumn{8}{|c|}{ Treatment of brain metastasis of NSCLC } \\
\hline Ma et al ${ }^{63}$ & II & Unselected $(n=2 I)$ & Unknown & $\begin{array}{l}\text { Gefitinib } \\
(250 \mathrm{mg})+\text { WBRT }\end{array}$ & 81.0 & $10.0 \mathrm{~m}$ & $13.0 \mathrm{~m}$ \\
\hline Wu et $\mathrm{al}^{62}$ & & Adenocarcinoma $(n=40)$ & Unknown & Gefitinib (250 mg) & 32.0 & $9.0 \mathrm{~m}$ & $15.0 \mathrm{~m}$ \\
\hline
\end{tabular}

Abbreviations: NSCLC, non-small-cell lung cancer; EGFR, epidermal growth factor receptor; ORR, overall response rate; PFS, progression-free survival; OS, overall survival; m, months; WBRT, whole brain irradiation; PD, progressive disease; IPASS, Iressa Pan Asia Study; M, mutation.

for advanced NSCLC patients who had progressed after systemic chemotherapy or were not suitable for systemic chemotherapy. Approximately 44,000 patients participated in this program. EAP was conducted during 2001-2007 in the People's Republic of China and brought in $\sim 1,600$ selected patients. Finally, the company did not process the formal statistical analysis of data. Retrospective analysis of the existing data (data deadline: March 31, 2006) indicated that patients had good response to gefitinib, with PFS being $>7$ months. A research that belonged to EAP analyzed the efficacy and safety of gefitinib in the treatment of recurrent advanced NSCLC. Among 103 patients, the ORR was $18.4 \%$ (19 of 103 ), the DCR was $51.5 \%$ (53 of 103 ), the median time to progression was 3 months (range: $0.2-40$ ), and the median survival time was 9.8 months (range: 0.5-51). AEs included skin rash, dry skin, diarrhea, and elevation of serum glutamate pyruvate transaminase and were usually mild. ${ }^{50}$

Another significant research taken by Peking Union Medical College Hospital evaluated the efficacy of gefitinib and EGFR mutation in a series of Chinese patients with pretreated advanced NSCLC. Ninety-eight consecutive patients were enrolled in the study between October 2002 and February 2004. Results showed an overall objective response rate of $31.6 \%$ and overall DCR of $67.3 \%$, which were associated with the histology type, sex, and smoking status in the subgroup analysis. The median PFS was $7.0 \pm 0.82$ months (95\% CI: 5.39-8.61), median OS was $12.0 \pm 1.87$ months (95\% CI: 8.33-15.67), and 1-year survival rate was 53.1\%. ${ }^{51}$ Both the response rate and DCR were higher than those in the Phase II trials described earlier but similar to the subgroup analysis results obtained in Japan. ${ }^{41}$ Geifitinib demonstrated significant antitumor activity in pretreated Chinese patients with advanced NSCLC. The authors proceeded EGFR mutation analysis as well, and 12 cases with EGFR mutations were detected, with four in-frame deletions in exon 19 (delE746-A750, delL747-P753 insS) and eight in exon 21. Mutation rates were higher in gefitinib responders, nonsmokers, patients with adenocarcinoma, and female patients. The PFS and OS for patients with mutation were significantly longer than those for patients without mutation.

\section{Gefitinib as first-line therapy}

Several large randomized controlled studies such as IPASS and First Signal showed noninferiority of gefitinib efficacy for chemonaive patients with advanced NSCLC compared with platinum-based chemotherapy. A further analysis of 376 Chinese people recruited from 20 medical centers in IPASS indicated that PFS did not significantly differ from that of the overall IPASS population (interaction test $P=0.427$ ), but 
PFS was numerically longer ( $\mathrm{HR}=0.79,95 \% \mathrm{CI}$ : 0.62-1.01, $P=0.065$; median PFS 6.8 months for both treatments) and ORR was significantly higher (ORR: $44.6 \%$ vs $29.8 \%$, odds ratio: $1.88,95 \% \mathrm{CI}: 1.22-2.89, P=0.004)$ for gefitinib than for carboplatin/paclitaxel. ${ }^{52}$ Another Chinese research similar to IPASS compared the clinical efficacy of gefitinib with that of paclitaxel/carboplatin in 51 ever-smokers and light exsmokers with advanced pulmonary adenocarcinoma receiving no prior chemotherapy or biological/immunological therapy. On the contrary, the study obtained different outcomes, showing that the median PFS was 4.2 vs 8.3 months $(P=0.422)$, ORR: $36.0 \%$ vs $42.3 \%(P=0.645)$, and median OS 14.4 vs 15.0 months $(P=0.290)$, comparing gefitinib with paclitaxel/carboplatin. ${ }^{53}$ This research indicated that first-line gefitinib should not be recommended for advanced NSCLC patients based only on clinical factors and for patients with unknown EGFR mutation status.

Numerous clinical studies demonstrated that active $E G F R$ mutation is an independent predictor for the effect of EGFR-TKI in advanced NSCLC. Another Chinese research that retrospectively analyzed 54 advanced NSCLC patients with positive EGFR mutation (in exons 19 or 21), who were given TKI drugs (gefitinib or erlotinib) as first-line therapy until disease progression, showed a significant benefit in ORR (90\%), median PFS (8.3 months), and median OS (19.5 months). Gefitinib had similar efficacy compared with erlotinib but higher safety. ${ }^{54}$

\section{Gefitinib as maintenance therapy}

At the same time as the first- and second-line therapeutic strategies were actively explored for advanced NSCLC, more attention was paid to maintenance therapy. Traditional treatment guidelines for unselected advanced NSCLC patients recommend four to six cycles of platinum-based doublet chemotherapy as standard first-line treatment, then to stop chemotherapy and enter the follow-up period. When a patient has a progressive disease, second-line therapy is recommended. However, most patients who receive first-line chemotherapy will very soon experience disease progression after initiating therapy, and the median survival is only 12-13 months. Moreover, as a study has reported, 50\%-60\% of patients with disease progression failed to accept secondline treatment owing to poor physical status. ${ }^{55}$ The role of maintenance therapy in patients who achieved tumor control with first-line chemotherapy has been gradually confirmed by series of investigations. On the basis of several research results, pemetrexed (for patients with tumors of nonsquamous histology) and erlotinib (for patients with stable disease) are now licensed for maintenance treatment of advanced NSCLC in Europe and the USA. ${ }^{56}$

Gefitinib is therefore a good candidate for maintenance treatment of advanced NSCLC because of its excellent antitumor efficacy and safety. The Phase III West Japan Thoracic Oncology Group maintenance trial (WJTOG0203) explored gefitinib maintenance therapy after platinum-doublet chemotherapy in $>600$ Japanese patients with advanced NSCLC. ${ }^{57}$ Patients receiving gefitinib had a statistically significant improvement in PFS compared with those remaining on platinum-doublet chemotherapy $(\mathrm{HR}=0.68,95 \% \mathrm{CI}$ : $0.57-0.80, P=0.001)$. Unfortunately, the primary end point of OS did not reach statistical significance.

INFORM was the first randomized, double-blind, placebo-controlled study conducted in Asian scholars to investigate the efficacy, safety, and tolerability of gefitinib in the maintenance setting in 296 patients with stage IIIb or IV NSCLC. ${ }^{58}$ Subjects were randomly assigned with the ratio of $1: 1$ to receive either gefitinib or placebo within 3-6 weeks after chemotherapy until progression or unacceptable toxic effects. PFS was significantly longer in the gefitinib group $(n=148)$ than in the placebo group $(n=148)$, and health-related quality-of-life improvement was also higher in the gefitinib group than that in the placebo group. However, similar to the WJTOG, OS did not reach statistical significance with 18.7 vs 16.9 months. The most common AEs of any grade were rash (73 [50\%] of 147 in the gefitinib group vs 14 [9\%] of 148 in the placebo group) and diarrhea (37 [25\%] vs 13 [9\%]). The outcome illustrated the efficacy and safety of gefitinib and affirmed the role of gefitinib maintenance therapy in Chinese patients with NSCLC.

\section{Gefitinib for brain metastasis of NSCLC}

Local recurrence and distant metastasis are the main causes for poor prognosis in NSCLC patients, and brain metastasis is one of the major causes. Approximately $40 \%$ of patients with NSCLC develop brain metastasis. ${ }^{59}$ Even with treatment, the prognosis of metastasis in the brain from NSCLC is very poor with a median OS 3-6 months. Animal studies have demonstrated a low concentration of [14C]-labeled gefitinib in normal rat brain and spinal cord ${ }^{60}$ and significant activity in brain tumor in a mouse model. ${ }^{61}$ In recent years, some published clinical studies also demonstrated gefitinib activity against brain metastases from NSCLC. Wu et al investigated the efficacy of gefitinib in palliative therapy for 40 patients who had histologically confirmed adenocarcinoma and brain metastases by radiological studies and had undergone chemotherapy previously. Results identified that gefitinib has 
promising activity for patients with brain metastasis from NSCLC, with ORR of $32 \%$, DCR of $77 \%$, PFS of 9.0 months, and OS of 15.0 months. ${ }^{62}$

Researchers also investigated the combination therapy of whole brain irradiation (WBRT) with targeted drugs for NSCLC brain metastasis and observed certain therapeutic effect. A Phase II clinical trial evaluated concomitant treatment with WBRT and gefitinib in patients with brain metastasis from NSCLC in a Chinese population. Totally, 21 patients recently diagnosed with histologically or cytologically confirmed NSCLC and measurable brain metastasis assessed by contrast-enhanced computed tomography scan or gadolinium-enhanced magnetic resonance imaging were involved in the study. ${ }^{63}$ The ORR (complete response + partial response) was $81 \%$ (95\% CI: 58\%-95\%) and the DCR (complete response + partial response + stable disease) was $95 \%$ (95\% CI: $76 \%-100 \%)$. The median PFS was 10.0 months (95\% CI: 7.5-12.5 months) and the median survival was 13.0 months (95\% CI: $8.2-$ 17.8 months), and a statistically significant improvement in the quality of life was attained with a questionnaire method. The most common AEs included rash (86\%) and diarrhea (43\%). Most side effects were grade II and well tolerated by supportive care. The results suggested that combination treatment of WBRT and gefitinib was well tolerated, with promising activity in a Chinese population with brain metastasis from NSCLC.

A retrospective research conducted in Taiwan assessed the outcome of brain metastasis from NSCLC treated by WBRT followed by gamma knife (GK), gefitinib, or the combination of GK and gefitinib; retrieving the records of 23,874 NSCLC patients with brain metastasis from the National Health Insurance Research Database (NHIRD) of Taiwan from 2004 to 2010 showed an extended survival in patients who received GK or gefitinib therapies in which the median survival periods from the time of diagnosis of brain metastasis with WBRT, WBRT + gefitinib, WBRT + GK, and WBRT + gefitinib + GK groups were $0.53,1.01$, 1.46 , and 2.25 years, respectively $(P<0.0001) .{ }^{64}$ This combination treatment for brain metastasis of lung cancer may be a new effective choice, although the exact curative effect needs other large placebo-controlled and randomized studies.

\section{Tolerability}

Gefitinib is generally well tolerated, and the most common toxic effects were diarrhea, rash, and acne, most of which were grade 1 or 2 (Common Toxicity Criteria). Drug-correlated
AEs observed in IDEAL I were skin rash, diarrhea, pruritus, asymptomatic elevations in liver transaminase levels, nausea, vomiting, and acne. Data reported in the ISEL and IDEAL I studies have shown that the types and incidence of gefitinib-induced AEs are similar between Asian and non-Asian patients, although the ISEL study reported more patients of Asian origin experiencing at least one AE with both gefitinib and placebo (97\% vs $82 \%$ for gefitinib; $87 \%$ vs $71 \%$ for placebo) and the frequency of grade 3 or $4 \mathrm{AEs}$ was also slightly higher (43\% vs 30\% for gefitinib; $36 \%$ vs $27 \%$ for placebo). ${ }^{65}$ Parts of the studies suggested that the incidence of diarrhea, asymptomatic elevations in liver transaminase levels, and nausea/vomiting was higher in Asian patients than that in non-Asian patients.

Data from the studies in the People's Republic of China have shown that the AE profile of gefitinib was similar to its profile from other large international trials. The most common AEs in INFORM study were skin rash, diarrhea, increased ALT and AST, and pruritus. Totally, 118 of 147 (80\%) patients receiving gefitinib had AEs, of which 98 were thought to be related to treatment. In the gefitinib group, nine (6\%) patients had an AE, leading to death, and three serious AEs, leading to death, were related to gefitinib - one due to interstitial lung disease (ILD), one due to lung infection, and one due to pneumonia. ${ }^{58}$

In the prospective study by Peking Union Medical College Hospital, AEs during treatment were diarrhea (40.9\%) and skin disorders (60.2\%), including dry skin and acne-like rash. ${ }^{51}$ The majority of these events were mild at Common Toxicity Criteria grades 1 and 2. Other AEs included nausea (6.1\%), oral ulcer (3.1\%), and elevation in hepatic enzymes $(2.0 \%)$. In the meta-analysis, 24 Chinese articles reported adverse reactions with the use of gefitinib, showing that AEs with a higher incidence were rash (47.11\%) and diarrhea (21.88\%). Twenty cases experienced elevation in liver transaminase levels and five cases suffered from ILD of which one case was grade IV and another died of severe ILD. Other adverse effects included nausea, fatigue, fever, oral ulcer, paronychia, pruritus, and joint pain, all with a very low incidence.

It is considered that the mechanism of dermatological toxicities involves nonspecific inhibition of EGFR signaling transduction by gefitinib in the skin, leading to skin keratinization, epifolliculitis, and loss of skin hydration. ${ }^{66}$ The occurrence of skin rash coinciding with symptom improvement reflects the body's response to EGFR inhibitors to some degree. Some trials suggested that patients with rash would acquire a better prognosis after gefitinib treatment. 
Dudek et al have shown that the presence of skin rash might be used as a predictor of gefitinib benefit, with the study result showing that patients who developed any degree of skin rash had prolonged time to disease progression with a median time of 6 months, while patients without a skin rash had a median time of 3 months. ${ }^{67}$ Another experiment found that gene polymorphism of ABCG2 (breast cancerresistance protein/mitoxantrone-resistance protein), which was demonstrated to be involved in transporting gefitinib, may be related to gefitinib toxicity. Tamura et al showed that $A B C G 234 \mathrm{G}>\mathrm{A}$ would be a useful predictor for grade 2 or worse skin rash. ${ }^{68}$ Lemos et al elucidated that patients carrying an $A B C G 2-15622 \mathrm{~T} / \mathrm{T}$ genotype or harboring at least one TT copy in the $A B C G 2(1143 \mathrm{C} / \mathrm{T}$ and $-15622 \mathrm{C} / \mathrm{T})$ haplotype developed significantly more grade $2 / 3$ diarrhea $(P<0.01){ }^{69}$

ILD is a rare but fatal adverse effect of gefitinib, with an unclear pathogenesis. Japanese researchers reported the first case of gefitinib-associated ILD. A 67-year-old man suffered progressive dyspnea on the eighth day of gefitinib administration and died from a pattern of diffuse alveolar damage 13 days after his first administration of gefitinib, despite administration of antibiotic therapy and high-dose of intravenous methylprednisolone $(2 \mathrm{~g} / \mathrm{d}) .{ }^{70}$ According to the data from Japan Labor Health and Welfare Organization, among the 17,500 advanced NSCLC patients who underwent gefitinib treatment, 219 cases developed gefitinib-associated ILD and acute lung injury, including 81 deaths. The incidence and mortality of fatal gefitinib-associated ILD were $0.44 \%$ and $0.12 \%$, respectively, in the USA, whereas in Japan, the respective rates were up to $1 \%-2 \%$ and $0.4 \%-0.5 \%$.

Several studies evaluating the correlation between ILD and gefitinib were case reports in mainland China. Researchers summarized nine case reports of ILD from 2003 to 2012 in mainland China, showing that ILD usually occurred in the period from the 24 th to the 42 nd day of gefitinib treatment, the earliest occurred within 2 days, and the last one occurred up to 3 months later. ${ }^{71}$ A retrospective study that analyzed 1,080 patients with advanced NSCLC in Taiwan who received at least one dose $(250 \mathrm{mg} / \mathrm{d})$ of gefitinib treatment showed that 25 patients were diagnosed with gefitinib-related ILD (incidence, 2.3\%). ${ }^{72}$ These findings raise the question of whether and why there are any higher risk factors for developing gefitinib-related ILD in Asians compared with non-Asians. More large-scale studies are needed. A study elucidated the risk factors for ILD in 3,166 Japanese patients with NSCLC during treatment with gefitinib and demonstrated that ILD was relatively common in patients with old age, smoking habits, preexisting ILD, or poor performance status. ${ }^{73}$

\section{Acquired resistance to EGFR-TKI}

Although impressive responses to gefitinib have been confirmed in NSCLC patients with EGFR mutations, tumor progression can occur as resistance develops. Most patients who initially respond to gefitinib and erlotinib eventually become resistant and experience progressive disease according to clinical observation. The most common mechanisms of EGFR-TKI resistance are the threonine-790 to methionine (T790M) point mutation, which accounts for about one-half of these cases with acquired resistance, and the MET gene amplification, accounting for another $20 \%{ }^{74,75}$ Kobayashi et al first discovered the T790M mutation after sequencing the EGFR gene of a patient with acquired resistance to gefitinib in $2005 .^{76}$ This was followed by a series of tremendous successes in this field. T790, located in the ATP-binding pocket, is named "the gatekeeper residue" as it determines the affinity of ATP-competitive EGFR-TKIs to EGFR-TK. Substitution of threonine 790 by methionine (T790M) increases the affinity of the ATP-binding site to EGFR and attenuates the binding efficacy of gefitinib and erlotinib consequently. ${ }^{77}$ MET receptor, a transmembrane TK encoded by proto-oncogene $M E T$, has a high-affinity ligand known as hepatocyte growth factor or scatter factor. ${ }^{78}$ The hepatocyte growth factor (HGF)/MET pathway is associated with tumor invasion, metastasis, and amplification. Engelman et $\mathrm{al}^{79}$ first found that focal amplification of the MET protooncogene caused gefitinib resistance by driving ERBB3 (HER3)-dependent activation of PI3K, a pathway believed to be specific to EGFR/ERBB family receptors. ${ }^{80}$

Because acquisition of the secondary resistance point mutation T790M increases the affinity of the ATP-binding site to EGFR and reduces the efficacy of ATP-competitive inhibitors, one strategy for preventing or overcoming EGFRTKI resistance could be to identify novel agents that can irreversibly block EGFR-TK via the formation of covalent bonds in the pocket of the catalytic site, including afatinib (BIBW 2992), HKI-272, and PF00299804. .9,81,82 Similar to the second-generation EGFR-TKI afatinib, these agents can potently and selectively suppress wild type and activated EGFR and HER2 mutants, including EGFR and HER2 inhibitor-resistant isoforms. ${ }^{83,84}$ A large Phase IIb/III study enrolled 585 patients with advanced metastatic NSCLC who had previously experienced treatment failures to erlotinib or gefitinib (or both) and one or two lines of chemotherapy. ${ }^{85}$ Although the primary end point (OS) showed no benefit from 
afatinib (afatinib vs placebo: 10.8 vs 12.0 months, $H R=1.08$, 95\% CI: $0.86-1.35, P=0.74)$, the afatinib group experienced a prolonged PFS (3.3 vs 1.1 months, $\mathrm{HR}=0.38,95 \% \mathrm{CI}$ : $0.31-0.48, P<0.0001)$ and confirmed better ORR (7\% vs $<1 \%$ ). A second strategy is targeting the HGF/MET pathway. For example, the MET TKI, tivantinib, is a non-ATPcompetitive small-molecule MET inhibitor. Given the good tolerability and potential ability of tivantinib both as singleagent therapy and in combination with erlotinib announced in several preclinical and Phase I clinical trials, a series of work have been carried out to evaluate its antitumor efficacy. ${ }^{86}$ Meanwhile, some other effective strategies for overcoming EGFR-TKI resistance such as drugs targeting HER3 pathway and $\mathrm{PI} 3 \mathrm{~K} / \mathrm{AKT} / \mathrm{mTOR}$ pathway are under research, and they are believed to be soon applied in the clinic.

\section{Conclusion}

A new era of advanced NSCLC therapy has been initiated by gefitinib, one of the first-generation TKIs developed and launched by AstraZeneca, providing a new effective treatment option for NSCLC patients. Based on several large international studies, gefitinib is now widely used in clinical practice. It is demonstrated that gefitinib significantly prolonged PFS, improved the quality of life, and was welltolerated when it was used as second- or third-line treatment for patients with advanced NSCLC who failed chemotherapy, and when it was used as first-line treatment or as a maintenance therapy following first-line chemotherapy for patients with EGFR mutation-positive advanced NSCLC. Some clinical characteristics such as Asian ethnicity, female sex, nonsmoking status, and adenocarcinoma have been associated with an enhanced benefit from gefitinib, which implies that gefitinib is particularly valuable to Oriental patients and now this drug is getting full attention from Chinese researchers. Both prospective and retrospective studies have verified the effective clinical responsiveness and safety of gefitinib treatment and established the essential role of gefitinib in Chinese patients with advanced NSCLC.

\section{Acknowledgment}

This work was supported by funding from Natural Science Foundation of China, number 81201787.

\section{Disclosure}

The authors report no conflicts of interest in this work.

\section{References}

1. Huang YC. Outdoor air pollution: a global perspective. J Occup Environ Med. 2014;56(suppl 10):S3-S7.
2. Chen W, Zheng R, Zhang S, Zhao P, Zeng H, Zou X. Report of cancer incidence and mortality in China, 2010. Ann Transl Med. 2014; 2(7):61.

3. Moschini I, Dell'Anna C, Losardo PL, Bordi P, D'Abbiero N, Tiseo M. Radiotherapy of non-small-cell lung cancer in the era of EGFR gene mutations and EGF receptor tyrosine kinase inhibitors. Future Oncol. 2015;11(16):2329-2342.

4. Wang X, Huang Z, Li H, Cai X. The short-term observation of Shenqifuzheng injection combined with NP chemotherapy in treating elder patients with advanced non-small cell lung cancer. Zhongguo Fei Ai Za Zhi. 2007;10(3):234-236.

5. Li CH, Liu MY, Liu W, Li DD, Cai L. Randomized control study of nedaplatin or cisplatin concomitant with other chemotherapy in the treatment of advanced non-small cell lung cancer. Asian Pac J Cancer Prev. 2014;15(2):731-736.

6. Reck M, Heigener DF, Mok T, Soria JC, Rabe KF. Management of nonsmall-cell lung cancer: recent developments. Lancet. 2013;382(9893): 709-719.

7. Roulston A, Wilkinson P, Haynes T, Campbell J. Complementary therapy: perceptions of older people with lung or colorectal cancer. Int JPalliat Nurs. 2013;19(7):333-339.

8. Pawuk LG, Schumacher JE. Introducing music therapy in hospice and palliative care: an overview of one hospice's experience. Home Healthc Nurse. 2010;28(1):37-44.

9. Quoix E, Zalcman G, Oster JP, et al; Intergroupe Francophone de Cancérologie Thoracique. Carboplatin and weekly paclitaxel doublet chemotherapy compared with monotherapy in elderly patients with advanced non-small-cell lung cancer: IFCT-0501 randomised, phase 3 trial. Lancet. 2011;378(9796):1079-1088.

10. Mok TS, Wu YL, Thongprasert S, et al. Gefitinib or carboplatin-paclitaxel in pulmonary adenocarcinoma. N Engl J Med. 2009;361(10):947-957.

11. Rosell R, Carcereny E, Gervais R, et al; Spanish Lung Cancer Group in collaboration with Groupe Français de Pneumo-Cancérologie and Associazione Italiana Oncologia Toracica. Erlotinib versus standard chemotherapy as first-line treatment for European patients with advanced EGFR mutation-positive non-small-cell lung cancer (EURTAC): a multicentre, open-label, randomised phase 3 trial. Lancet Oncol. 2012; 13(3):239-246.

12. Kwak EL, Bang YJ, Camidge DR, et al. Anaplastic lymphoma kinase inhibition in non-small-cell lung cancer. N Engl J Med. 2010; 363(18):1693-1703.

13. Forsythe B, Faulkner K. Overview of the tolerability of gefitinib (IRESSA) monotherapy: clinical experience in non-small-cell lung cancer. Drug Saf. 2004;27(14):1081-1092.

14. Pan ZY, Jiang ZS, Ouyang HQ. Study of the methylation patterns of the EGFR gene promoter in non-small cell lung cancer. Genet Mol Res. 2015;14(3):9813-9820.

15. Witton CJ, Reeves JR, Going JJ, Cooke TG, Bartlett JM. Expression of the HER1-4 family of receptor tyrosine kinases in breast cancer. J Pathol. 2003;200(3):290-297.

16. Downward J, Yarden Y, Mayes E, et al. Close similarity of epidermal growth factor receptor and v-erb-B oncogene protein sequences. Nature. 1984;307(5951):521-527.

17. Hanahan D, Weinberg RA. The hallmarks of cancer. Cell. 2000;100(1): 57-70.

18. Schlessinger J. Common and distinct elements in cellular signaling via EGF and FGF receptors. Science. 2004;306(5701):1506-1507.

19. Ribeiro Gomes J, Cruz MR. Combination of afatinib with cetuximab in patients with EGFR-mutant non-small-cell lung cancer resistant to EGFR inhibitors. Onco Targets Ther. 2015;8:1137-1142.

20. Yarden Y. The EGFR family and its ligands in human cancer, signaling mechanisms and therapeutic opportunities. Eur J Cancer. 2001; 37(suppl 4):S3-S8.

21. Vastag B. Research unveils the 'who' and 'why' of gefitinib. J Natl Cancer Inst. 2004;96(18):1352-1354.

22. Lynch TJ, Bell DW, Sordella R, et al. Activating mutations in the epidermal growth factor receptor underlying responsiveness of non-small-cell lung cancer to gefitinib. N Engl J Med. 2004;350(21):2129-2139. 
23. Huang SF, Liu HP, Li LH, et al. High frequency of epidermal growth factor receptor mutations with complex patterns in non-small cell lung cancers related to gefitinib responsiveness in Taiwan. Clin Cancer Res. 2004;10(24):8195-8203.

24. Murray S, Dahabreh IJ, Linardou H, Manoloukos M, Bafaloukos D, Kosmidis P. Somatic mutations of the tyrosine kinase domain of epidermal growth factor receptor and tyrosine kinase inhibitor response to TKIs in non-small cell lung cancer: an analytical database. J Thorac Oncol. 2008;3(8):832-839.

25. Metro G, Crinò L. Advances on EGFR mutation for lung cancer. Transl Lung Cancer Res. 2012;1(1):5-13.

26. Qin BM, Chen X, Zhu JD, Pei DQ. Identification of EGFR kinase domain mutations among lung cancer patients in China: implication for targeted cancer therapy. Cell Res. 2005;15(3):212-217.

27. Mu XL, Li LY, Zhang XT, et al. Gefitinib-sensitive mutations of the epidermal growth factor receptor tyrosine kinase domain in Chinese patients with non-small cell lung cancer. Clin Cancer Res. 2005;11(12): 4289-4294.

28. Swaisland H, Laight A, Stafford L, et al. Pharmacokinetics and tolerability of the orally active selective epidermal growth factor receptor tyrosine kinase inhibitor ZD1839 in healthy volunteers. Clin Pharmacokinet. 2001;40(4):297-306.

29. Ding JF, Zhong DF. Clinical pharmacokinetics of small molecule tyrosine kinase inhibitors. Yaо Хие Хие Bao. 2013;48(7):1080-1090.

30. Swaisland HC, Smith RP, Laight A, et al. Single-dose clinical pharmacokinetic studies of gefitinib. Clin Pharmacokinet. 2005;44(11):1165-1177.

31. Cantarini MV, McFarquhar T, Smith RP, Bailey C, Marshall AL. Relative bioavailability and safety profile of gefitinib administered as a tablet or as a dispersion preparation via drink or nasogastric tube: results of a randomized, open-label, three-period crossover study in healthy volunteers. Clin Ther. 2004;26(10):1630-1636.

32. Cantarini MV, Bailey CJ, Collins B, Smith RP. The relative bioavailability of gefitinib administered by granular formulation. Cancer Chemother Pharmacol. 2008;62(2):203-208.

33. Bergman E, Forsell P, Persson EM, et al. Pharmacokinetics of gefitinib in humans: the influence of gastrointestinal factors. Int J Pharm. 2007;341(1-2):134-142.

34. Tanović A, Alfaro V. Gefitinib: current status in the treatment of nonsmall cell lung cancer. Drugs Today (Barc). 2004;40(10):809-827.

35. Cohen MH, Williams GA, Sridhara R, et al. United States food and drug administration drug approval summary: gefitinib (ZD1839; Iressa) tablets. Clin Cancer Res. 2004;10(4):1212-1218.

36. Li J, Zhao M, He P, Hidalgo M, Baker SD. Differential metabolism of gefitinib and erlotinib by human cytochrome P450 enzymes. Clin Cancer Res. 2007;13(12):3731-3737.

37. Ranson M, Hammond LA, Ferry D, et al. ZD1839, a selective oral epidermal growth factor receptor-tyrosine kinase inhibitor, is well tolerated and active in patients with solid, malignant tumors: results of a phase I trial. J Clin Oncol. 2002;20(9):2240-2250.

38. Herbst RS, Maddox AM, Rothenberg ML, et al. Selective oral epidermal growth factor receptor tyrosine kinase inhibitor ZD1839 is generally welltolerated and has activity in non-small-cell lung cancer and other solid tumors: results of a phase I trial. J Clin Oncol. 2002;20(18):3815-3825.

39. Fukuoka M, Yano S, Giaccone G, et al. Multi-institutional randomized phase II trial of gefitinib for previously treated patients with advanced non-small-cell lung cancer(The IDEAL 1 Trial) [corrected]. J Clin Oncol. 2003;21(12):2237-2246.

40. Kris MG, Natale RB, Herbst RS, et al. Efficacy of gefitinib, an inhibitor of the epidermal growth factor receptor tyrosine kinase, in symptomatic patients with non-small cell lung cancer. A randomized trial. J Am Med Assoc. 2003;290(16):2149-2158.

41. Tamura K, Fukuoka M. Gefitinib in non-small cell lung cancer. Expert Opin Pharmacother. 2005;6(6):985-993.

42. Thatcher N, Chang A, Parikh P, et al. Gefitinib plus best supportive care in previously treated patients with refractory advanced nonsmall-cell lung cancer: results from a randomized, placebo-controlled, multicenter study (Iressa survival evaluation in lung cancer). Lancet. 2005;366(9496):1527-1537
43. Chang A, Parikh P, Thongprasert S. Gefitinib (IRESSA) in patients of Asian origin with refractory advanced non-small cell lung cancer: subset analysis from the ISEL study. $J$ Thorac Oncol. 2006;1(8): $847-855$.

44. Kim ES, Hirsh V, Mok T, et al. Gefitinib versus docetaxel in previously treated non-small-cell lung cancer (INTEREST): a randomised phase III trial. Lancet. 2008;372(9652):1809-1818.

45. Takeda M, Nakagawa K. Role of EGFR monoclonal antibodies in the management of non-small cell lung cancer. Curr Cancer Drug Targets. 2015;15(9):792-802.

46. Yang JC, Kang JH, Mok T, et al. First-line pemetrexed plus cisplatin followed by gefitinib maintenance therapy versus gefitinib monotherapy in East Asian patients with locally advanced or metastatic non-squamous non-small cell lung cancer: a randomised, phase 3 trial. Eur J Cancer. 2014;50(13):2219-2230.

47. Soria JC, Wu YL, Nakagawa K, et al. Gefitinib plus chemotherapy versus placebo plus chemotherapy in EGFR-mutation-positive non-small-cell lung cancer after progression on first-line gefitinib (IMPRESS): a phase 3 randomised trial. Lancet Oncol. 2015;16(8):990-998.

48. Tan YK, Wee TC, Koh WP, et al. Survival among Chinese women with lung cancer in Singapore: a comparison by stage, histology and smoking status. Lung Cancer. 2003;40(3):237-246.

49. Guan ZZ, Zhang L, Li LY, et al. Efficacy of gefitinib on Chinese patients with locally advanced or metastatic non-small cell lung cancer: a clinical trial. Ai Zheng. 2005;24(8):980-984.

50. Huang H, Zhang Y, Zhao HY, et al. Analysis of the efficacy and safety of gefitinib in the treatment of recurrent advanced non-small cell lung cancer in an expanded access program (EAP). Zhonghua Zhong Liu Za Zhi. 2009;31(2):148-151.

51. Zhang XT, Li LY, Mu XL, et al. The EGFR mutation and its correlation with response of gefitinib in previously treated Chinese patients with advanced non-small-cell lung cancer. Ann Oncol. 2005; 16(8):1334-1342.

52. Wu YL, Chu DT, Han B, et al. Phase III, randomized, open-label, first-line study in Asia of gefitinib versus carboplatin/paclitaxel in clinically selected patients with advanced non-small-cell lung cancer: evaluation of patients recruited from mainland China. Asia Pac J Clin Oncol. 2012;8(3):232-243.

53. Lou N, Yang J, Yan H, et al. Efficacies of gefitinib versus paclitaxel/ carboplatin for patients with advanced pulmonary adenocarcinoma. Zhonghua Yi Xue Za Zhi. 2014;94(30):2337-2341.

54. Li J, Qu L, Wei X, et al. Clinical observation of EGFR-TKI as a firstline therapy on advanced non-small cell lung cancer. Zhongguo Fei Ai Za Zhi. 2012;15(5):299-304.

55. Stinchcombe TE, Socinski MA. Treatment paradigms for advanced stage non-small cell lung cancer in the era of multiple lines of therapy. J Thorac Oncol. 2009;4(2):243-250.

56. Tucker $S$. The role of pemetrexed in second-line chemotherapy for advanced non-small cell lung cancer. Curr Drug Targets. 2010;11(1):58-60.

57. Takeda K, Hida T, Sato T, et al. Randomized phase III trial of platinumdoublet chemotherapy followed by gefitinib compared with continued platinum-doublet chemotherapy in Japanese patients with advanced non-small-cell lung cancer: results of a west Japan thoracic oncology group trial (WJTOG0203). J Clin Oncol. 2010;28(5):753-760.

58. Zhang L, Ma S, Song X, et al; INFORM Investigators. Gefitinib versus placebo as maintenance therapy in patients with locally advanced or metastatic non-small-cell lung cancer (INFORM; C-TONG 0804): a multicenter, double-blind randomized phase 3 trial. Lancet Oncol. 2012; 13(5):466-475.

59. Rizzi A, Tondini M, Rocco G, et al. Lung cancer with a single brain metastasis: therapeutic options. Tumori. 1990;76(6):579-581.

60. McKillop D, Hutchison M, Partridge EA, et al. Metabolic disposition of gefitinib, an epidermal growth factor receptor tyrosine kinase inhibitor, in rat, dog and man. Xenobiotica. 2004;34(10):917-934.

61. Heimberger AB, Learn CA, Archer GE, et al. Brain tumors in mice are susceptible to blockade of epidermal growth factor receptor (EGFR) with the oral, specific, EGFR-tyrosine kinase inhibitor ZD1839 (iressa). Clin Cancer Res. 2002;8(11):3496-3502. 
62. Wu C, Li YL, Wang ZM, Li Z, Zhang TX, Wei Z. Gefitinib as palliative therapy for lung adenocarcinoma metastatic to the brain. Lung Cancer. 2007;57(3):359-364.

63. Ma S, Xu Y, Deng Q, Yu X. Treatment of brain metastasis from nonsmall cell lung cancer with whole brain radiotherapy and gefitinib in a Chinese population. Lung Cancer. 2009;65(2):198-203.

64. Lin $\mathrm{CH}$, Hsu KH, Chang SN, et al. Increased survival with the combination of stereotactic radiosurgery and gefitinib for non-small cell lung cancer brain metastasis patients: a nationwide study in Taiwan. Radiat Oncol. 2015;10:127.

65. Jiang H. Overview of gefitinib in non-small cell lung cancer: an Asian perspective. Jpn J Clin Oncol. 2009;39(3):137-150.

66. Lacouture ME. Mechanisms of cutaneous toxicities to EGFR inhibitors. Nat Rev Cancer. 2006;6(10):803-812.

67. Dudek AZ, Kmak KL, Koopmeiners J, Keshtgarpour M. Skin rash and bronchoalveolar histology correlates with clinical benefit in patients treated with gefitinib as a therapy for previously treated advanced or metastatic non-small cell lung cancer. Lung Cancer. 2006;51(1):89-96.

68. Tamura M, Kondo M, Horio M, et al. Genetic polymorphisms of the adenosine triphosphate-binding cassette transporters (ABCG2, ABCB1) and gefitinib toxicity. Nagoya J Med Sci. 2012;74(1-2):133-140.

69. Lemos C, Giovannetti E, Zucali PA, et al. Impact of ABCG2 polymorphisms on the clinical outcome and toxicity of gefitinib in non-small-cell lung cancer patients. Pharmacogenomics. 2011;12(2):159-170.

70. Okamoto I, Fujii K, Matsumoto M, et al. Diffuse alveolar damage after ZD1839 therapy in a patient with non-small cell lung cancer. Lung Cancer. 2003;40(3):339-342.

71. Zhang X, Li H, Zhu M, Zhang Y. Re-administration of gefitinib following diffuse interstitial lung disease in a patient with advanced lung adenocarcinoma: a case report and review of the literature. Oncol Lett. 2015;9(5):2419-2421.

72. Chang SC, Chang CY, Chang SJ, et al. Gefitinib-related interstitial lung disease in Taiwanese patients with non-small-cell lung cancer. Clin Lung Cancer. 2013;14(1):55-61.

73. Kudoh S, Kato H, Nishiwaki Y, et al; Japan Thoracic Radiology Group. Interstitial lung disease in Japanese patients with lung cancer: a cohort and nested case-control study. Am J Respir Crit Care Med. 2008;177(12): $1348-1357$.

74. Balak MN, Gong YX, Riely GJ, et al. Novel D761Y and common secondary T790M mutations in epidermal growth factor receptor mutant lung adenocarcinomas with acquired resistance to kinase inhibitors. Clin Cancer Res. 2006;12(21):6494-6501.
75. Suda K, Onozato R, Yatabe Y, Mitsudomi T. EGFR T790M mutation: a double role in lung cancer cell survival? J Thorac Oncol. 2009;4(1):1-4.

76. Kobayashi S, Boggon TJ, Dayaram T, et al. EGFR mutation and resistance of non-small-cell lung cancer to gefitinib. N Engl J Med. 2005; 352(8):786-792.

77. Yun CH, Mengwasser KE, Toms AV, et al. The T790M mutation in EGFR kinase causes drug resistance by increasing the affinity for ATP. Proc Natl Acad Sci US A. 2008;105(6):2070-2075.

78. Nakamura T, Nishizawa T, Hagiya M, et al. Molecular cloning and expression of human hepatocyte growth factor. Nature. 1989;342(6248): 440-443.

79. Engelman JA, Zejnullahu K, Gale CM, et al. PF00299804, an irreversible pan ErbB inhibitor, is effective in lung cancer models with EGFR and HER2 mutations that are resistant to gefitinib. Cancer Res. 2008;67(24):11924-11932.

80. Rosell R, Taron M, Sanchez JJ, Paz-Ares L. Setting the benchmark for tailoring treatment with EGFR tyrosine kinase inhibitors. Future Oncol. 2007;3(3):277-283.

81. Kwak EL, Sordella R, Bell DW, et al. Irreversible inhibitors of the EGFR receptor may circumvent acquired resistance to gefitinib. Proc Natl Acad Sci U S A. 2005;102(21):7665-7670.

82. Wong KK. HKI-272 in non-small cell lung cancer. Clin Cancer Res. 2007;13(15 pt 2):S4593-S4596.

83. Li D, Ambrogio L, Shimamura T, et al. BIBW2992, an irreversible EGFR/HER2 inhibitor highly effective in preclinical lung cancer models. Oncogene. 2008;27(34):4702-4711.

84. Yoshikawa S, Kukimoto-Niino M, Parker L, et al. Structural basis for the altered drug sensitivities of non-small cell lung cancer-associated mutants of human epidermal growth factor receptor. Oncogene. 2013; 32(1):27-38.

85. Miller VA, Hirsh V, Cadranel J, et al. Afatinib versus placebo for patients with advanced, metastatic non-small cell lung cancer after failure of erlotinib, gefitinib, or both, and one or two lines of chemotherapy (LUX-Lung 1): a phase 2b/3 randomised trial. Lancet Oncol. 2012; 13(5):528-538

86. Rosen LS, Senzer N, Mekhail T, et al. A phase I dose-escalation study of tivantinib (ARQ 197) in adult patients with metastatic solid tumors. Clin Cancer Res. 2011;17(24):7754-7764.
OncoTargets and Therapy

\section{Publish your work in this journal}

OncoTargets and Therapy is an international, peer-reviewed, open access journal focusing on the pathological basis of all cancers, potential targets for therapy and treatment protocols employed to improve the management of cancer patients. The journal also focuses on the impact of management programs and new therapeutic agents and protocols on

\section{Dovepress}

patient perspectives such as quality of life, adherence and satisfaction The manuscript management system is completely online and includes a very quick and fair peer-review system, which is all easy to use. Visit http://www.dovepress.com/testimonials.php to read real quotes from published authors. 